\title{
Predictors of weight loss are different in men and women after sleeve gastrectomy
}

\author{
John Roger Andersen, $\mathrm{PhD}{ }^{1,2}$, Eivind Aadland, $\mathrm{PhD}^{2}$, Roy Miodini \\ Nilsen, $\mathrm{PhD}^{3}$ and Villy Våge, $\mathrm{PhD}^{1}$. \\ ${ }^{1}$ Department of Surgery, Førde Central Hospital, Førde, Norway. ${ }^{2}$ Department of Health \\ Studies, Sogn og Fjordane University College, Førde, Norway. ${ }^{3}$ Centre for Clinical \\ Research, Haukeland University Hospital, Bergen, Norway. \\ Correspondence. John Roger Andersen, johnra@ @isf.no
}

\begin{abstract}
Introduction - Weight loss following bariatric surgery varies according to patient factors before the intervention. However, whether predictors of weight loss differ between men and women is, to our knowledge, unknown. We therefore aimed to investigate baseline predictors for overall weight loss and identify potential sex-specific baseline predictors in bariatric surgery patients. Methods - In this prospective cohort study, 160 patients (117 women and $43 \mathrm{men}$ ) who underwent sleeve gastrectomy were followed for two years. Weight loss was defined as percent excess body mass index loss (\%EBMIL). To investigate whether \%EBMIL differed between men and women, we included all two-way interactions with sex by incorporating the product term sex and predictors using multiple linear regression analysis. Results - The overall mean \pm standard deviation of \%EBMIL after two years was $78.3 \pm 23.5$. Predictors for lower \%EBMIL in a regression model with no interactions were female sex $(\mathrm{P}=0.003)$, higher body mass index before surgery $(\mathrm{P}=0.001)$ and non-smoking $(\mathrm{P}=0.029)$. When examining sexspecific predictors for \%EBMIL, higher age $(\mathrm{P}=0.027)$ and not having diabetes $(\mathrm{P}=0.007)$ predicted lower \%EBMIL in men. In women, unemployment $(\mathrm{P}=0.006)$ and anxiety and/or depression $(\mathrm{P}=0.009)$ predicted lower \%EBMIL. Conclusion - This study suggests that weight loss and predictors for weight loss two years after sleeve gastrectomy are sex-specific. These findings may be useful for the surgical strategy used to treat these patients.
\end{abstract}

Keywords. Obesity, Predictors, Weight loss, Bariatric surgery, Sleeve gastrectomy 


\section{Introduction}

Successful weight loss after bariatric surgery is often defined as greater than $50 \%$ excess weight loss; however, in the long term, more than $30 \%$ of patients fail to achieve this goal $[1,2]$. Unsuccessful weight loss is associated with lack of resolution of comorbidities and reduced quality of life [2-4]. Thus, identifying preoperative predictive factors for weight loss might be useful in developing strategies to improve weight loss following bariatric surgery [5]. However, the results from studies evaluating potential predictors of weight loss after bariatric surgery are inconclusive, and further studies with new approaches are needed $[1,5]$.

We hypothesized that predictors of weight loss after bariatric surgery differ according to sex. If this hypothesis is confirmed, then treatment could be tailored accordingly. However, whether predictors of weight loss after bariatric surgery differ according to sex is, to our knowledge, unknown. What is known and prompted us to perform this study is that men and women differ greatly in terms of fat storage and metabolism [6]. Interestingly, when men and women are asked to explain their inability to lose weight after bariatric surgery, they also seem to emphasize different causes [7]. Thus, there may be "hidden differences" between men and women in terms of weight loss that have been unreported in the literature. Such sex differences could perhaps explain some of the inconsistent results founds in previous studies $[1,5]$.

Therefore, the aim of this study was to explore potential preoperative predictors (e.g., demographical and clinical variables) of weight loss after sleeve gastrectomy (SG). We hypothesized that predictors for weight loss differed between men and women.

\section{Material and methods}

Patients who were accepted for SG at Førde Central Hospital were invited to participate in a prospective cohort study. The criteria for having surgery included $\mathrm{BMI} \geq 40.0$ or $35.0-39.9$ with obesity-related comorbidities, age $18-60$, no alcohol or drug problems, no active psychosis and failure to lose weight via other methods. Data were collected at baseline (the preoperative evaluation), preoperatively (the day before surgery), and one and two years after surgery. The study database was approved by the Norwegian Data Inspectorate and conforms to the principles outlined in of the Declaration of Helsinki. 


\section{The SG procedure}

Prior to SG, patients participated in a one-day course on obesity and bariatric surgery, which was followed by individual consultation with a bariatric surgeon and other health personnel if needed. Preoperative advice included smoking cessation, increased physical activity and weight loss. The operation was performed laparoscopically through six ports, and all vessels at the greater curvature from the pylorus to the cardia were divided by LigaSure (Covidien). The stomach was resected along a 32 French bougie from $2-3 \mathrm{~cm}$ proximal to the pylorus to the cardia using a Tri-Stapler (Covidien). Postoperative advice included a low carbohydrate high protein diet, the intake of one multivitamin tablet daily, a high frequency of water intake and physical activity. Controls and data collection occurred at the outpatient clinic 3,12 and 24 months postoperatively.

\section{Outcome}

The outcome variable percent excess body mass index loss (\%EBMIL) (from baseline to two-year follow-up) was calculated using the following formula: 100(follow-up BMI-25/beginning BMI-25)*100) [8]. Body weight was measured in light clothing without shoes to the nearest 0.1 kilogram. Height was measured in a standing position without shoes to the nearest 0.01 meter.

\section{Predictors}

Predictors were selected based on previous research [1, 9], theoretical considerations and variable availability. The following predictors were included: Patient age (years), sex, marital status (married/cohabitation versus unmarried/cohabitation), education level ( $<13$ versus $\geq 13$ years of education), employment status (unemployed versus employed), time from baseline (preoperative consultation) to surgery ( $\geq 12$ months versus $<12$ months), BMI at baseline, \%EBMIL from baseline to surgery ( $\leq 5 \%$ versus $>5 \%$ ), smoking (nonsmoking versus smoking), diabetes (no versus yes), anxiety and/or depression (no versus yes) and musculoskeletal pain (no versus yes). Due to collinearity, we combined having anxiety and/or depression into one variable. Comorbidities were considered to be present only if the patient's general physician confirmed the diagnosis and the patient was on documented treatment.

\section{Statistics}

Statistical analyses were performed using the IBM SPSS version 20 software for Windows. $\mathrm{P}$ values were 2 -sided, and values $<0.05$ were considered statistically significant. Continuous variables were described as the mean and standard deviation 
(SD), whereas categorical variables were described using the number and percentage. To examine sex-specific changes in BMI over time, we used generalized estimating equations for continuous data. Intra-individual correlation was accounted for using the exchangeable variance-covariance structure. Because of multiple testing, the $\mathrm{P}$ values were corrected using the Sidak method.

Simple and multiple linear regression models were constructed using SPSS (using the general linear model-univariate module) to study baseline predictors for $\%$ EBMIL after two years. Unstandardized regression coefficients and 95\% confidence intervals were reported. To investigate whether predictors of \%EBMIL differed between men and women, we included all two-way interactions with sex by incorporating the product term sex and predictor in multivariable models. To obtain sex-specific estimates for predictors that had a significant interaction effect, we ran multiple regression models twice, with men and women coded differently ( 0 versus 1 and 1 versus 0 ). The clinical significance (effect size) was estimated for each statistically significant predictor. For continuous variables, we computed the difference in predicted \%EBMIL corresponding to a 2-SD difference in predictor variables [10]. For categorical variables, we computed the difference in predicted $\%$ EBMIL relative to the reference category. These differences were divided by the SD of the \%EBMIL in the entire sample at two years follow-up. These SD units or effect sizes were evaluated against the standard criteria proposed by Cohen: Trivial $(<0.2)$, small $(0.2$ to $<0.5)$, moderate $(0.5$ to $<0.8)$, and large $(\geq 0.8)[11]$.

\section{Results}

By the two-year follow-up, we had data from 117 women and 43 men (93\% followup rate) (Table 1 and Figure 1). The BMI significantly changed between all-time points for the entire cohort $(\mathrm{P}<0.001)$, with the exception of between the one- and two-year follow-ups $(\mathrm{P}=0.999)$. The mean $\mathrm{BMI} \pm \mathrm{SD}$ at preoperative evaluation and at the two-year follow-up was $46.8 \pm 6.9$ and $30.2 \pm 5.7$, respectively. The mean \pm SD of \%EBMIL at the two-year follow-up was $78.3 \pm 23.5$ for the entire cohort and $86.9 \pm 23.9$ and $75.1 \pm 22.6$ for men and women, respectively (P for sex differences $=0.004)$.

Multiple regression analysis showed that female sex, higher BMI and non-smoking were significant predictors for lower \%EBMIL (Table 2). Interaction analysis showed that the variables age, employment status, diabetes and anxiety and/or depression significantly predicted \%EBMIL differently in men and women (Table 3). In men, increasing age and not having a diagnosis of diabetes were significant predictors for lower \%EBMIL, while unemployment and a diagnosis of anxiety and/or depression were significant predictors for lower \%EBMIL in women (Table 
3). The predictive values of the other variables were unaffected by the inclusion of interaction terms (data not shown). The effect sizes for the statistically significant predictors ranged from 0.44 to 0.86 (Table 4). We also performed secondary analysis with \%EBMIL at the one-year follow-up as a dependent variable. The directions of the associations were essentially similar but generally much weaker. The only statistically significant findings were that higher BMI at baseline predicted lower \%EBMIL and employment status had a different predictive effect on $\%$ EBMIL according to sex (data not shown).

\section{Discussion}

The principal finding of this study is that age, employment status, diabetes and anxiety and/or depression predicted \%EBMIL differently for men and women. When we did not consider sex differences, we found that only female sex, higher preoperative BMI and non-smoking were significant predictors of lower \%EBMIL. The gender differences in predictors of weight loss did only manifest themselves two years after surgery.

Using multiple regression analysis not including sex interactions, we observed that women had a lower \%EBMIL than men. In general, the literature is inconsistent on this issue [9]. However, as found in this study, a 12.1 percentage point difference in $\%$ EBMIL is clinically relevant. We also observed that 18 of the 117 women (16\%), but only 1 of the 44 men $(2.3 \%)$, had a $\%$ EBMIL $<50 \%$. If these results can be replicated, one hypothesis is that SG may differently influence satiety in men and women.

Our observation that higher BMI at baseline was associated with lower \%EBMIL in both sexes is in accordance with a large body of research [1]. This finding is best explained by the fact that high BMI is an indicator of underlying causes of obesity [6]. Thus, a higher BMI indicates that more radical surgery is likely to be needed if a high \%EBMIL is to be obtained.

We also observed that non-smoking was associated with lower \%EBMIL for both sexes. This association may be related to the effects of smoking on eating behavior and metabolism [12]. However, the limited literature is mixed on whether smoking is associated with greater weight loss after bariatric surgery [9]. Even if there is a small but positive association between smoking and weight loss, the effect of smoking equals having a BMI > 40 in terms of risk for lost life years [13], and this risk of smoking should be communicated to patients.

For the non-significant findings in this study, the lack of association between preoperative weight loss and \%EBMIL after two years is perhaps most interesting. 
The results in the literature are mixed [1], and we believe that positive associations between preoperative weight loss and weight loss after surgery most likely fade with time [14]. However, we still encourage preoperative weight loss to reduce operative risk.

We will now discuss sex interactions.

First, the observation that men with diabetes had a particularly high \%EBMIL is interesting because several studies have shown that having diabetes is associated with lower weight loss after bariatric surgery [9]. Diabetes was not a predictor for $\%$ EBMIL in women in this study, but the P-value was borderline significant. A few studies have suggested that men with diabetes may benefit more from behavioral weight reduction programs than women with diabetes, a finding that has been explained by the fact that men with diabetes appear to comply better with treatment regimens $[15,16]$. Thus, it is possible that men with diabetes who had bariatric surgery ate less and/or had more physical activity. Nevertheless, our observations suggest that the negative association between diabetes and weight loss after bariatric surgery in the literature may only apply to women.

Second, we observed that increasing age predicted lower \%EBMIL in men. Thus, our observations suggest that the negative association between age and weight loss reported in the literature [9] is not independent of sex, and could perhaps be related to declining physical activity as one ages, which may have a particular negative effect on weight loss in men [17]. Thus, men may need to be informed that they are at increased risk for lower weight loss as they age.

Third, we found that unemployment predicted lower \%EBMIL in women. The interaction between sex and employment status could be related to differences in work-related behavior. If more men than women travel as a part of their work, working men may thus adhere less to a healthy lifestyle [18]. It is also possible that women who are employed are more physically active than unemployed women. Thus, encouraging and helping patients get back to work may be important for maximizing weight loss if one is able to maintain healthy behaviors.

Finally, we found that women with a diagnosis of anxiety and/or depression had a lower \%EBMIL than those without these diagnoses, while no such association was observed for men. Again, the literature of predictors of weight loss is mixed [1], and our observations suggest that not taking sex interactions into account may to some extent explain why. The literature suggests that women with obesity tend to have worse self-reported mental health than men with obesity [19]. If this difference is associated with postoperative eating behavior, it may explanation our observations. Unfortunately, we did not have data that allowed us to explore this issue. 
The main strengths of this study are the low attrition rate and that the predictor selection for the regression models was based on previous research and included tests for sex interactions. To our knowledge, this is the first study of predictors of \%EBMIL after SG that investigates sex interactions for weight loss after bariatric surgery. This study also has limitations that should be addressed. First, we had no data on diet or physical activity after SG. Thus, we do not know whether our set of preoperative predictors was associated with the health behaviors that SG was intended to influence. Furthermore, our set of predictors was not exhaustive, and the male sample size was relatively small, reflecting the fact that fewer men than women choose bariatric surgery. However, the BMI at baseline and standard deviation for \%EBMIL after two years were quite similar in men and women. Nevertheless, the results in the male group should be interpreted with caution. Finally, although our study had a longer follow-up than many of the published studies [1], weight regain is more common in the longer term [2]. Therefore, studies with long-term follow-up are clearly needed.

In conclusion, our findings suggest that predictors of weight loss after SG differ for men and women two years after surgery. Further studies should be done to test the external validity of our findings. Thus, we recommend examining sex interactions in future studies on predictors of weight loss after bariatric surgery.

\section{Conflict of interest disclosure statement}

No conflict of interest.

\section{Literature}

1. Livhits M, Mercado C, Yermilov I, Parikh JA, Dutson E, Mehran A, Ko CY, Gibbons MM. Preoperative Predictors of Weight Loss Following Bariatric Surgery: Systematic Review. Obes Surg. Aug 2011;22(1):70-89.

2. Biron S, Hould FS, Lebel S, Marceau S, Lescelleur O, Simard S, Marceau P. Twenty years of biliopancreatic diversion: what is the goal of the surgery? Obes Surg. Feb 2004;14(2):160-4.

3. Sjöström CD, Lystig T, Lindroos AK. Impact of weight change, secular trends and ageing on cardiovascular risk factors: 10-year experiences from the SOS study. Int J Obes (Lond). Nov 2011;35(11):1413-20.

4. Karlsson J, Taft C, Ryden A, Sjöström L, Sullivan M. Ten-year trends in healthrelated quality of life after surgical and conventional treatment for severe obesity: the SOS intervention study. Int J Obes Aug 2007;31(8):1248-61. 
5. van Hout GC, Verschure SK, van Heck GL. Psychosocial predictors of success following bariatric surgery. Obes Surg. Apr 2005;15(4):552-60.

6. Power M, Schulkin J. The evolution of obesity. Baltimore, The Johns Hopkins University Press, 2009.

7. Hwang KO, Childs JH, Goodrick GK, Aboughali WA, Thomas EJ, Johnson CW, $\mathrm{Yu}$ SC, Bernstam EV. Explanations for unsuccessful weight loss among bariatric surgery candidates. Obes Surg. Oct 2009;19(10):1377-83.

8. Baltasar A, Deitel M, Greenstein RJ. Weight loss reporting. Obes Surg. Jun 2008;18(6):761-2.

9. Adams ST, Salhab M, Hussain ZI, Miller GV, Leveson SH. Roux-en-Y gastric bypass for morbid obesity: what are the preoperative predictors of weight loss? Postgrad Med J. Jul 2013;89(1053):411-6.

10. Gelman A. Scaling regression inputs by dividing by two standard deviations. Stat Med. Jul 2008;27(15):2865-73.

11. Cohen J. Statistical Power Analysis for the Behavioral Sciences. 1 ed. New York, Academic Press, 1988.

12. Chiolero A, Faeh D, Paccaud F, Cornuz J. Consequences of smoking for body weight, body fat distribution, and insulin resistance. Am J Clin Nutr. Apr 2008;87(4):801-9.

13. Whitlock G, Lewington S, Sherliker P, Clarke R, Emberson J, Halsey J, Qizilbash N, Collins R, Peto R. Body-mass index and cause-specific mortality in 900000 adults: collaborative analyses of 57 prospective studies. Lancet. Mar 28 2009;373(9669):1083-96.

14. Harnisch MC, Portenier DD, Pryor AD, Prince-Petersen R, Grant JP, DeMaria EJ. Preoperative weight gain does not predict failure of weight loss or co-morbidity resolution of laparoscopic Roux-en-Y gastric bypass for morbid obesity. Surg Obes Relat Dis. May-Jun 2008;4(3):445-50.

15. Wadden TA, Neiberg RH, Wing RR, Clark JM, Delahanty LM, Hill JO, Krakoff J, Otto A, Ryan DH, Vitolins MZ. Four-year weight losses in the Look AHEAD study: factors associated with long-term success. Obesity (Silver Spring). Oct 2011;19(10):1987-98. 
16. Heitzmann CA, Kaplan RM, Wilson DK, Sandler J. Sex differences in weight loss among adults with type II diabetes mellitus. J Behav Med. Apr 1987;10(2):197211.

17. Levine JA. Nonexercise activity thermogenesis (NEAT): environment and biology. Am J Physiol Endocrinol Metab. May 2004;286(5):E675-85.

18. Wandel M, Roos G. Work, food and physical activity. A qualitative study of coping strategies among men in three occupations. Appetite. Feb 2005;44(1):93102.

19. Kolotkin RL, Crosby RD, Williams GR. Health-related quality of life varies among obese subgroups. Obes Res. Aug 2002;10(8):748-56. 


\section{Tables and figures}

Table 1. Patient characteristics

\begin{tabular}{llll}
\hline Characteristics & All $(\mathrm{N}=160)$ & Women $(\mathrm{n}=117)$ & Men $(\mathrm{n}=43)$ \\
\hline Age, years; mean \pm sd. & $40.4 \pm 11.1$ & $37.7 \pm 11.1$ & $42.5 \pm 10.9$ \\
Married/cohabitation, $\mathrm{n}(\%)$ & $91(56.9)$ & $71(60.7)$ & $20(46.5)$ \\
Education ( $\geq 13$ years; $\mathrm{n}(\%)$ & $41(25.6)$ & $33(28.2)$ & $8(18.6)$ \\
Employed, $\mathrm{n}(\%)$ & $103(64.4)$ & $77(65.8)$ & $26(60.5)$ \\
Waiting time*, $\geq 12$ months, $\mathrm{n}(\%)$ & $12(7.5)$ & $11(9.4)$ & $1(2.3)$ \\
Body mass index, mean \pm sd. & $46.8 \pm 6.9$ & $46.8 \pm 6.4$ & $46.6 \pm 8.2$ \\
Preop. EBMIL, >5\%, $\mathrm{n}(\%)$ & $60(37.5)$ & $42(35.9)$ & $18(41.9)$ \\
Smoking, $\mathrm{n}(\%)$ & $31(19.4)$ & $23(19.7)$ & $8(18.6)$ \\
Musculoskeletal pain, $\mathrm{n}(\%)$ & $72(45.0)$ & $51(43.6)$ & $21(48.8)$ \\
Diabetes, $\mathrm{n}(\%)$ & $32(20.0)$ & $20(17.1)$ & $12(27.9)$ \\
Mental disorder, $\mathrm{n}(\%)$ & $36(22.5)$ & $26(22.2)$ & $10(23.3)$ \\
\hline
\end{tabular}

Note: EBMIL: excess body mass index loss. ${ }^{*}$ : Waiting time is defined as the time from preoperative evaluation to surgery. 
Table 2. Linear regression analysis with percent excess body mass index loss after two years as the dependent variable $(\mathrm{N}=160)$

\begin{tabular}{|c|c|c|c|c|}
\hline & Simple regression & & Multiple regressi & \\
\hline Dependent variables & $\mathrm{B}(95 \% \mathrm{Cl})$ & P-value & $\mathrm{B}(95 \% \mathrm{Cl})$ & P-value \\
\hline Age (years) & $-0.2(-0.5,0.1)$ & 0.260 & $-0.2(-0.5,0.2)$ & 0.318 \\
\hline $\begin{array}{l}\text { Sex } \\
\text { (ref. men) }\end{array}$ & $-11.8(-19.9,-3.7)$ & 0.004 & $-12.1(-20.0,-4.2)$ & 0.003 \\
\hline $\begin{array}{l}\text { Married/cohabitation (ref. } \\
\text { no) }\end{array}$ & $0.8(-6.7,8.2)$ & 0.841 & $-0.3(-7.4,6.8)$ & 0.926 \\
\hline $\begin{array}{l}\text { Education } \\
\text { (ref. }<13 \text { years) }\end{array}$ & $-3.3(-11.7,5.1)$ & 0.436 & $-3.4(-11.6,4.7)$ & 0.411 \\
\hline $\begin{array}{l}\text { Employed } \\
\text { (ref. no) }\end{array}$ & $-7.1(-14.7,0.5)$ & 0.065 & $-5.0(-12.8,2.8)$ & 0.204 \\
\hline $\begin{array}{l}\text { Waiting time }{ }^{*} \\
\text { (ref. >12 months) }\end{array}$ & $-6.8(-20.7,7.1)$ & 0.336 & $-3.1(-16.3,10.2)$ & 0.648 \\
\hline $\begin{array}{l}\text { BMI } \\
\text { (units at baseline) }\end{array}$ & $-1.1(-1.6,-0.6)$ & $<0.001$ & $-0.9(-1.4,-0.4)$ & 0.001 \\
\hline $\begin{array}{l}\% \text { EBMIL } \\
\text { (ref. } \leq 5 \% \text { ) }\end{array}$ & $-0.3(-7.9,7.3)$ & 0.945 & $3.3(-4.2,10.9)$ & 0.385 \\
\hline $\begin{array}{l}\text { Smoking } \\
\text { (ref. no) }\end{array}$ & $13.3(4.3,22.4)$ & 0.004 & $10.3(1.1,19.5)$ & 0.029 \\
\hline $\begin{array}{l}\text { Diabetes } \\
\text { (ref. no) }\end{array}$ & $1.4(7.8,10.6)$ & 0.764 & $0.9(-8.0,9.7)$ & 0.848 \\
\hline $\begin{array}{l}\text { Mental disorder } \\
\text { (ref. no) }\end{array}$ & $-8.0(-16.7,0.8)$ & 0.073 & $-7.0(-15.5,1.5)$ & 0.105 \\
\hline $\begin{array}{l}\text { Musculoskeletal pain (ref. } \\
\text { no) }\end{array}$ & $-0.0(-7.4,7.3)$ & 0.991 & $-0.7(-7.7,6.4)$ & 0.854 \\
\hline
\end{tabular}

Note: Waiting time is defined as the time from preoperative evaluation to surgery. Adjusted $R^{2}=15.6 \%$. 
Table 3. Gender-specific linear multiple regression analysis with percent excess body mass index loss after two years as the dependent variable $(\mathrm{N}=160)$

\begin{tabular}{lll}
\hline Predictors & $\mathrm{B}(95 \% \mathrm{Cl})$ & P-value \\
\hline Gender x age interaction & $\mathrm{NA}$ & 0.047 \\
Age (years) & & 0.027 \\
$\quad$ Men & $-0.8(-1.3,-0.8)$ & 0.925 \\
Women & $0.2(-0.4,0.4)$ & 0.001 \\
Gender x employment interaction & $\mathrm{NA}$ & \\
Employed (ref. no) & & 0.054 \\
Men & & 0.006 \\
Women & $-13.1(-26.3,0.3)$ & 0.002 \\
Gender x diabetes interaction & $12.4(3.6,21.1)$ & \\
Diabetes (ref. no) & $\mathrm{NA}$ & 0.007 \\
$\quad$ Men & & 0.092 \\
Women & & 0.017 \\
Gender x Anxiety and/or depression interaction & $20.2(5.5,34.9)$ & \\
Anxiety and/or depression (ref. no) & $-9.1(-19.7,1.5)$ & 0.250 \\
Men & $\mathrm{NA}$ & 0.009 \\
Women & & \\
\hline
\end{tabular}

Note: Only sex-specific estimates from significant gender interactions are presented. The estimates are adjusted for the baseline values for age, gender, marital status, education, waiting time, body mass, preoperative percent excess weight loss, smoking, diabetes, anxiety and /or depression and musculoskeletal pain. NA: not applicable. 
Table 4. Effect sizes of statistically significant predictors of \%EBMIL

\begin{tabular}{lc}
\hline Predictors & Effect size \\
\hline General predictors & -0.51 \\
Sex (ref. men) & -0.58 \\
Body mass index (per 2 SD) & 0.44 \\
Smoking (ref. no) & \\
& \\
Predictors only in men & -0.72 \\
Higher age (per 2 SD) & 0.86 \\
Diabetes (ref. no) & \\
& \\
Predictors only in women & 0.53 \\
Employed (ref.no) & -0.55 \\
Having anxiety and/or depression (ref.no) & \\
\hline
\end{tabular}

Note: For the continuous variables, we computed the difference in predicted percent excess body mass index loss (\%EBMIL) corresponding to a 2-SD difference in the predictor variables. For categorical variables, we computed the difference in predicted \%EBMIL relative to the reference category. These differences were divided by 23.5 (the SD of the \%EBMIL in the entire sample at two years follow-up). Effect sizes were judged against the standard criteria proposed by Cohen: trivial $(<0.2)$, small $(0.2$ to $<0.5)$, moderate $(0.5$ to $<0.8)$, and large $(\geq 0.8)$ 


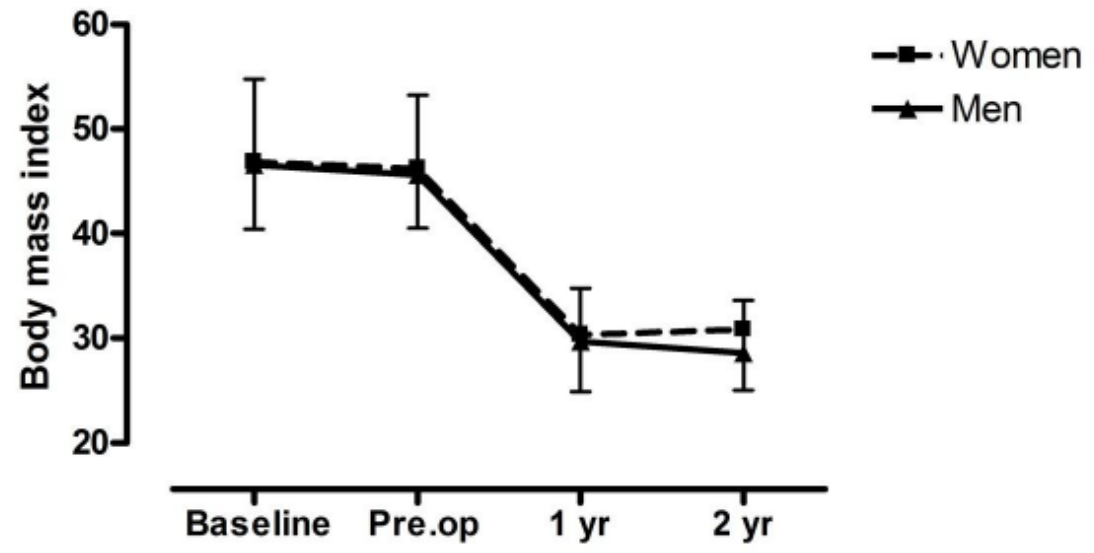

Figure 1. Means and standard deviations for body mass index in men ( $=43)$ and women $(n=117)$. Pre.op: preoperative. 1 y: 1 year. 2 y: 2 years. 